\title{
A CMOS Image Sensor With Reconfigurable Resolution For Energy Harvesting Applications
}

\author{
Chao Shi, Man Kay Law, and Amine Bermak \\ Department of Electronic and Computer Engineering \\ The Hong Kong University of Science and Technology \\ Clear Water Bay, Kowloon, Hong Kong SAR, China \\ Email:\{eecshi, eeml, and eebermak\}@ust.hk
}

\begin{abstract}
In this paper, we propose a CMOS image sensor with reconfigurable resolution for energy harvesting applications such as video sensor networks. Depending on the available energy, the spatial resolution of the imager can be adaptively reconfigured to save energy for other units on the sensor node. In contrast to early attempts of energy harvesting image sensor, we propose an asynchronous sensor in which the photodetector itself within the pixel can be used as an energy harvesting device, so that the total available energy will be increased. Low power operation is achieved since the time-to-first spike (TFS) pixel only fires once per frame. Utilizing address-event-representation, the imager can make efficient use of the output bandwidth. System architecture and operation are discussed together with the simulation results. Measurement results for a test structure fabricated in standard $0.35 \mu \mathrm{m}$ CMOS process are also provided. A system model is developed to illustrate the effectiveness of the proposed approach.
\end{abstract}

\section{INTRODUCTION}

Modern portable imaging systems are expected to consume higher power due to increased image resolution, improved processing features, as well as increased signal-to-noise ratio (SNR). However, portable systems requiring extended system lifetime demand low power operation. With highly efficient batteries, this dilemma might be resolved. Unfortunately, the amount of energy available is still quite low. It is expected that next generation image sensors will consume less than $1 \mathrm{~mW}$ to support the demand for continuous power reduction in mobile devices [1][2], while supporting increasing resolution and complexity. This problem is even more critical for applications with limited accessibility such as sensor networks, biomedical implants and embedded micro-sensors, since human intervention for energy replenishment implies higher operational cost.

Apart from resorting to low power sensor architectures, researchers begin to adopt energy harvesting techniques to deal with limited energy available on-chip [3]. Recently, a number of energy scavenging approaches using photodiodes in standard CMOS technology have been proposed [4][5]. In [5], the concept of Self-Powered Sensor (SPS) was first introduced. An additional photodiode, referred to as Power Generation Photodiode (PGPd) is used to harvest energy from the incident light for the sensor reset operation and in-pixel amplifier, hence reducing the sensor power dissipation from the regular power supply. Though this work shows excellent potential in terms of energy harvesting for CMOS image sensors, it still suffers several drawbacks, such as an inefficient use of the photosensitive area, and long charge-up period under poor illumination.

In this paper, we propose an energy harvesting CMOS image sensor architecture using Time-to-First-Spike (TFS) pixel together with Address-Event-Representation (AER) readout scheme and reconfigurable resolution. The TFS pixel exhibits asynchronous behavior such that pixels with brighter illumination will fire first and subsequently are configured into energy harvesting mode. Highly illuminated pixel will be contributing power at an earlier stage, and hence increasing the overall power generation efficiency. Moreover, depending on the available energy, the spatial resolution of the image senor can be reconfigured. Though image quality might be degraded, the energy consumption can be scaled down dramatically, both for the sensor array and peripheral circuits. This paper is organized as follows: Section II presents the proposed energy harvesting TFS pixel. Section III discusses the imager architecture. Measurement results and system model are provided in section IV. A conclusion is drawn in section V.

\section{Proposed Energy Harvesting TFS Pixel}

Fig. 1 shows the proposed TFS pixel with parasitic capacitances at critical nodes also illustrated. The pixel consists of two photodiodes and 18 transistors. Pd operates as the photodetector, while PGPd works as the energy harvesting device. Transistors MP2-4 and MN2-5 form the current feedback event generator. The handshaking communication is realized by transistors MP6-8. The other transistors operate as switches to control the operation of the pixel. The $V_{\text {power }}$ node is charged up by PGPd and Pd, and serves as the power supply for the pixel. $V_{\mathrm{N}}$ is the voltage at the sensing node of the photodetector, and $V_{\mathrm{GEN}}$ is the output voltage of the event generator. $V_{\mathrm{ASR}}$ is the internal control signal used to enable/disable the connection between the sensing node of the photodetector and $V_{\text {power }}$ node. Reset, Refresh, $\overline{\text { RowAck, }}$ and ColAck are peripheral control signals, and RowReq and ColReq are the request signals generated from the pixel to the row and column arbitration trees. $\overline{\text { RowAck }}$ and ColAck are the signals generated by the arbitration block and sent back to the pixel to acknowledge the handshaking process.

Image capture process is invoked by refreshing the $V_{\mathrm{ASR}}$ node. Integration process is then initiated by resetting the pixel. The light falling onto the photodiode will charge the 


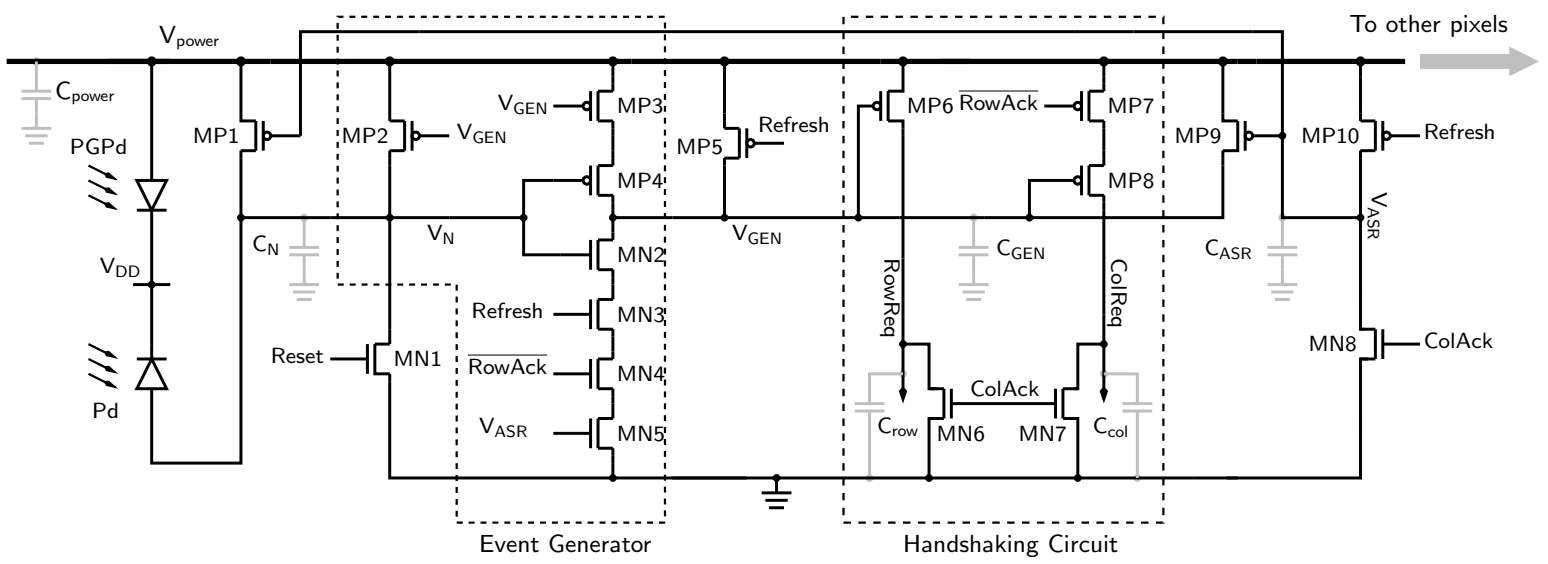

Fig. 1. Schematic of the proposed pixel. The pixel consists of a photodector (Pd), a power generation photodiode (PGPd), an event generator, and a handshaking communication circuit. Critical parasitic capacitances are also illustrated.

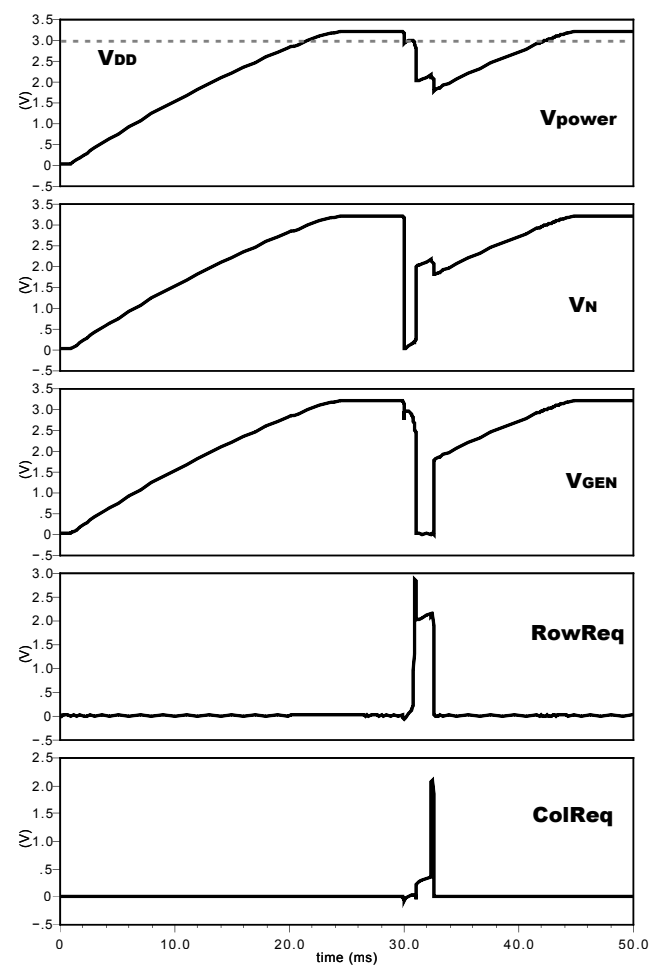

Fig. 2. Transient simulation results for the pixel under office lighting.

sensing node and $V_{\mathrm{N}}$ will increase linearly. Once this voltage reaches the threshold voltage of the event generator, a spike corresponding to the time to reach the threshold, will be generated. The transition of $V_{\mathrm{GEN}}$ from high to low turns on MP2, thus connecting the anode of Pd to the $V_{\text {power }}$ node. This enables Pd to contribute power to the $V_{\text {power }}$ node, and makes it available to other pixels. As MP8 has already been turned on by $V_{\mathrm{GEN}}$, ColReq will be immediately sent out when the pixel is acknowledged by $\overline{R o w A c k}$. Finally, column acknowledgement signal ColAck is sent back and turns on MN6-8 to remove the request signals. Meanwhile, $V_{\mathrm{ASR}}$ is also pulled down to switch on MP1 and MP9. $V_{\text {GEN }}$ is therefore pulled up to $V_{\text {power }}$, clearing the event and turning off MP2. Meanwhile, as MP1 is turned on, Pd continues to provide extra power for other pixels. It can be observed that once the pixel event is generated, the photodetector, Pd, continuously harvests energy from the incident light, and contributes the harvested charge to the operation of other pixels through the $V_{\text {power }}$ node.

A prototype pixel is designed in AMIS $0.35 \mu \mathrm{m}$ CMOS digital process, and it takes up an area of $33.4 \times 33.4 \mu \mathrm{m}^{2}$, with PGPd and Pd occupying $450 \mu \mathrm{m}^{2}$ and $150 \mu \mathrm{m}^{2}$, respectively. To ensure robust handshaking communication, amplitudes for request signals should be larger than $2 \mathrm{~V}$ with a supply of $3 \mathrm{~V}$. The transient simulation results for the proposed pixel under 500 lux light condition (corresponds to an office light setting) are illustrated in Fig. 2, which validate the proposed design. The variation of $V_{\text {power }}$ indicates the charge dissipation of the pixel. The amplitude for the ColReq signal is about $2.1 \mathrm{~V}$, which meets the design requirement. In our proposed pixel, $13.45 \%$ of the area is used for photosensing, but the total energy harvesting area can be extended to $53.78 \%$ once the photodetector is configured to harvest energy. Compared with [5], in which only $1 / 14$ of the pixel area is used for photosensing and $30.6 \%$ is used for energy harvesting, our approach shows better utilization of the silicon area.

\section{AER-BASEd IMAGER with ReConfigurable RESOLUTION}

The architecture of the imager utilizing the proposed energy harvesting TFS-based pixel array is shown in Fig. 3. Unlike other digital pixel image sensors, in which the illumination information is digitized and stored using a pixel-level memory, the imager here employs an event based readout method. Pixel generated events are placed on a shared bus and arbitration is executed at both row and column levels to multiplex the two dimensional information into a single output channel. This type of readout methodology is generally referred to as "Address Event Representation", and is now gaining increased attention [6]. 


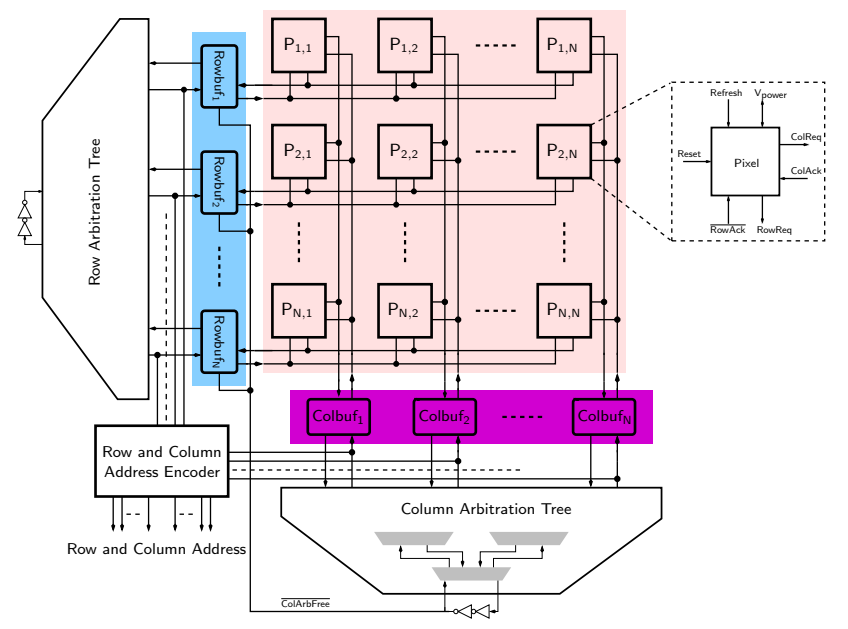

Fig. 3. Image sensor architecture overview. The sensor includes a TFS pixel array, column and row buffers and arbitration trees, as well as column and row address encoders.

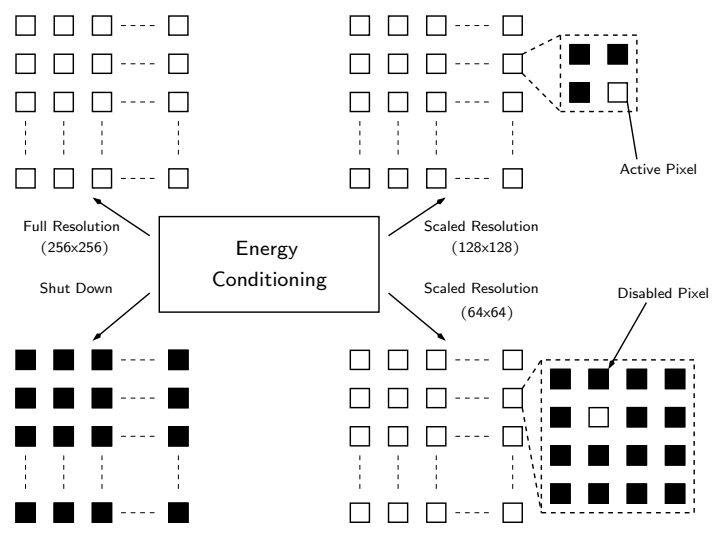

Fig. 4. Illustration of the proposed reconfigurable resolution approach.

In energy harvesting based sensor network applications, the energy available varies since the ambient illumination level varies considerably. In order to operate in worse case scenarios for which the available energy is limited, the sensor should be able to scale down its energy consumption, for critical situations. In this work, we propose to acquire this feature by adjusting the imager's resolution depending on the level of energy available. Fortunately, the spatial resolution of image sensors can be easily controlled by simple peripheral logic. Though image quality might be degraded at a reduced resolution, the energy consumption can be scaled down dramatically, both at the sensor array level and for peripheral circuits. The operation principle is illustrated in Fig. 4. Depending on the available energy, the energy conditioning unit determines a suitable spatial resolution for the image senor. When the available energy is low, the spatial resolution is reduced. The disabled pixels, which are not involved in event generation, are continuously harvesting energy from the environment, thus increasing the total available energy. The peripheral control

method is explained in Fig. 5 with Reset signal as an example. With limited additional control logic and routing overhead, the spatial resolution can be quite easily reconfigured.

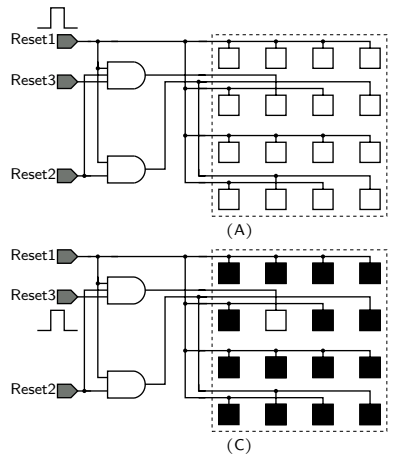

Fig. 5. Control method for reconfigurable resolution scheme: (A) full resolution $(256 \times 256)$, (B) scaled resolution $(128 \times 128)$, (C) scaled resolution $(64 \times 64)$, and $(\mathrm{D})$ shuntdown.

\section{EXPERIMENTAL RESUlTS AND SySTEM MODEL}

\section{A. Test Structure and Measurement Results}

In order to test the proposed scheme, a test structures was implemented using 0.35- $\mu \mathrm{m}$ AMIS digital CMOS technology. The test structure is fabricated to illustrate the functionality of the power generation capability of PGPd, as well as the dynamics of the voltage drop of $V_{\text {power }}$ over time. Since the handshaking communication is just a simple charge sharing process, a simplified version of the proposed pixel is designed, which includes the energy harvesters and the event generator. An increase in size of PGPd and $\mathrm{Pd}$ is required so that $C_{\mathrm{PGPd}}$ and $C_{\mathrm{Pd}}$ dominate over the extra capacitance introduced by the buffer stage. The test structure employs standard $\mathrm{p}+/ \mathrm{n}$-well photodiodes for both Pd and PGPd, with areas of $20 \times 20 \mu \mathrm{m}^{2}$, and $100 \times 100 \mu \mathrm{m}^{2}$, respectively.

The schematic of the fabricated test structure is shown in Fig. 6. This test structure consists of a photodetector Pd and PGPd, a reset transistor, a current feedback event generator, and buffers. $V_{\text {power }}$ is measured at the output of the buffer at node $V_{\text {out } 1}$, and the generated event is read out through $V_{\text {out2 }}$. The Reset signal frequency was set at $25 \mathrm{~Hz}$. The power generation process is illustrated in Fig. 7, together with the event generation process. After reset, $V_{\text {out } 1}$ begins to drop due to the charge dissipation of the event generator. Once the event is generated, $V_{\text {out1 }}$ starts to recover since there is no other source of power consumption. The transition of $V_{\text {out2 }}$ from low to high reveals that the event is successfully generated. The peak-to-peak voltage for $V_{\text {out } 1}$ was measured at about $1.44 \mathrm{~V}$. This value represents the amount of energy dissipated during the event generation process. Once the event is generated, $V_{\text {power }}$ is charged up again.

\section{B. System Model}

In order to model the proposed energy harvesting scheme at the behavioral level, as well as to illustrate the benefits 


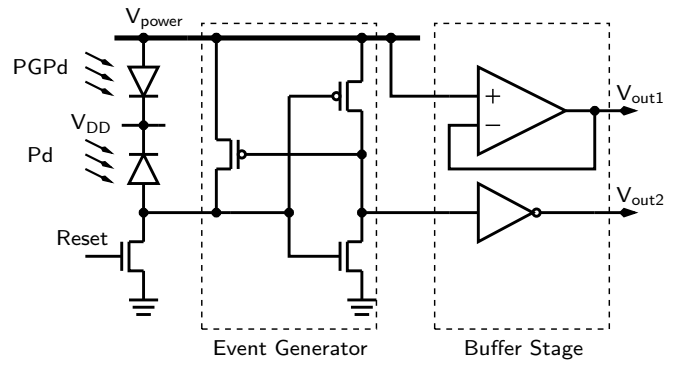

Fig. 6. Schematic of the test structure.

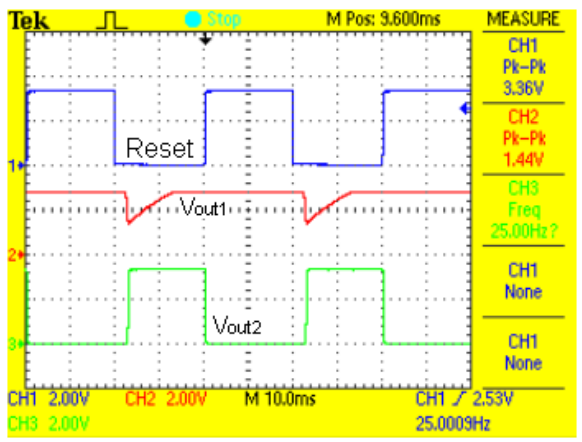

Fig. 7. Measured power generation process and event generation process in the test structure under 500lux illumination.

brought by the reconfigurable resolution scheme, a certain image distribution has to be assumed with given average intensity levels. Therefore, MATLAB is utilized to convert an input image into a set of firing times, which are then used to evaluate the amount of energy harvested from the array. This is a system level simulation and any transistor level simulator will require a tremendous amount of time to simulate the whole array. Sample $256 \times 2568$-bit images are used to test the proposed energy harvesting scheme at the system level. Simulation results of one frame for "Lena" image are illustrated in Fig. 8. Image capture process starts at $10 \mathrm{~ms}$. $V_{\text {power }}$ drops quickly at the beginning due to the increased number of fired pixels consuming charge for event generation, charging internal nodes, and handshaking communication. As more pixels have been readout, $V_{\text {power }}$ stops to drop and begins to recover. The $V_{\text {power }}$ variation and total frame time are summarized in TABLE. I. With a scaled resolution, the

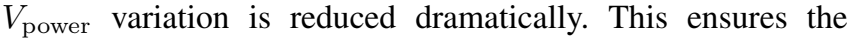
robust operation of sensor when the available energy is low.

\section{CONCLUSION}

In this paper, an AER based CMOS image sensor with reconfigurable resolution utilizing a TFS energy harvesting pixel is proposed. Compared with early attempts of energy harvesting image sensors, our scheme uses the photodetector itself for power generation, which leads to better utilization of the photosensitive area. The asynchronous characteristic

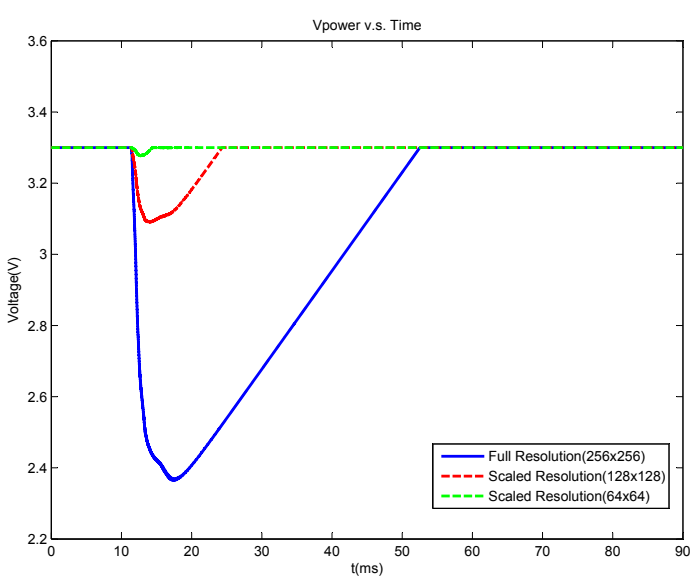

Fig. 8. Dynamics of $V_{\text {power }}$ for arrays of full resolution $(256 \times 256)$ and scaled resolution $(128 \times 128)$ and $(64 \times 64)$.

TABLE I

$V_{\text {power }}$ VARiation and Total Frame Time For Different Spatial RESOLUTIONS

\begin{tabular}{|c|c|c|}
\hline & $V_{\text {power }}$ Variation( $(\mathrm{V})$ & Total Frame Time(ms) \\
\hline Full resolution $(256 \times 256)$ & 0.93 & 42.5 \\
\hline Scaled Resolution $(128 \times 128)$ & 0.21 & 15.2 \\
\hline Scaled Resolution $(64 \times 64)$ & 0.023 & 12.3 \\
\hline
\end{tabular}

leads to an improved energy generation scheme. The resolution reconfigurability enables the sensor to scale down its power consumption when the available energy is low. Both simulation and measurement results are proposed to illustrate the proposed concept.

\section{ACKNOWLEDGMENT}

The authors would like to acknowledge the support of the Research Grant Council of Hong Kong under CERG Grant Ref: 610507.

\section{REFERENCES}

[1] C. Xu, W. Zhang, W. H. Ki, and M. Chan, "A 1.0-V VDD CMOS Active Pixel Sensor With Complementary Pixel Architectures and Pulse Width Modulation Fabricated with a $0.25 \mu \mathrm{m}$ CMOS Process," IEEE J. SolidState Circuits., Vol. 37, No. 12, pp. 1853-1859, Dec. 2002.

[2] H. S. Wong, R. T. Chang, E. Crabbe, and P. D. Agneelo, "CMOS Active Pixel Image Sensors Fabricated Using a $1.8 \mathrm{~V} 0.25 \mu \mathrm{m}$ CMOS Technology," IEEE Trans. Electron Devices, Vol. 45, No. 4, pp. 889-894, Apr. 1998.

[3] M. Rahimi, et al, "Studying the Feasibility of Energy Harvesting in a Mobile Sensor Network," in Proc. IEEE ICRA, 2003, pp. 19-24.

[4] N. Guilar, T. Kleeburg, A. Chen, D. Yankelevich, and R. Amirtharajah "Integrated Solar Energy Harvesting and Storage," IEEE Trans. Very Large Scale Integr. (VLSI) Syst., Vol. 17. No. 5, pp. 627-637, May. 2009.

[5] A. Fish, S. Hamami, and O. Yadid-Pecht, "Self-Powered Active Pixel Sensors For Ultra Low-Power Applications," in Proc. IEEE Int. Symp. Circuits Syst. (ISCAS), 2005, pp. 5310-5313.

[6] S. Chen, and A. Bermak, "Arbitrated Time-to-First Spike CMOS Image Sensor With On-Chip Histogram Equalization", IEEE Trans. Very Large Scale Integr. (VLSI) Syst., Vol. 15, No. 3, pp. 346-357, Mar. 2007. 